\title{
Sexualidade e Religiosidade: uma revisão integrativa de literatura
}

\author{
Sexuality and Religiosity: an integrative literature review \\ Sexualidad y Religiosidad: una revisión integradora de la literatura
}

Recebido: 01/02/2021 | Revisado: 09/02/2021 | Aceito: 12/02/2021 | Publicado: 19/02/2021

\author{
Helena Moraes Cortes \\ ORCID: https://orcid.org/0000-0001-8538-8400 \\ Universidade Federal do Recôncavo da Bahia, Brasil \\ E-mail: helenamoraescortes@gmail.com \\ Andréia Vanessa Carneiro Morais \\ ORCID: https://orcid.org/0000-0001-5051-8228 \\ Universidade Federal do Recôncavo da Bahia, Brasil \\ E-mail: andreiavmorais14@gmail.com \\ Luanna Carolyne Silva De Lacerda \\ ORCID: https://orcid.org/0000-0003-3788-9029 \\ Universidade Federal do Recôncavo da Bahia, Brasil \\ E-mail: luannadelacerda@gmail.com \\ Renata Oliveira Santos \\ ORCID: https://orcid.org/0000-0002-3865-1496 \\ Universidade Federal do Recôncavo da Bahia, Brasil \\ E-mail: s.oliveirarenata1@gmail.com \\ Paula Hayasi Pinho \\ ORCID: https://orcid.org/0000-0001-8922-0699 \\ Universidade Federal do Recôncavo da Bahia, Brasil \\ E-mail: paulahpinho@gmail.com
}

\begin{abstract}
Resumo
A influência da religião nas práticas sexuais humanas tem sido estudada ao longo dos anos. Objetivou-se conhecer a produção científica nacional e internacional acerca da relação entre sexualidade e religiosidade de 2012 a 2017. Tratase de uma revisão integrativa de literatura. Utilizou-se as bases de dados Scielo, Pubmed e BVS. 167 artigos foram encontrados, sendo 40 selecionados seguindo as recomendações da estratégia Prisma. Os resultados apontaram 3 categorias temáticas, a saber: a religiosidade como fator de risco ou proteção na sexualidade durante o processo saúde/doença, a religiosidade e a sexualidade no ciclo vital e em grupos populacionais, e a religiosidade e a sexualidade na prevenção de HIV/AIDS. Concluiu-se que a religião pode interferir no processo saúde-doença; estudála é necessário para a compreensão do indivíduo em diversas esferas e para que propostas de intervenções possam ser implementadas de acordo com as necessidades do sujeito.
\end{abstract}

Palavras-chave: Religiosidade; Sexualidade; Processo saúde/doença; Cuidado; Saúde.

\begin{abstract}
The influence of religion on human sexual practices has been studied over the years. The objective was to know the national and international scientific production about the relationship between sexuality and religiosity from 2012 to 2017. This is an integrative literature review. Scielo, Pubmed and BVS databases were consulted and the 167 articles were found, 40 of them being selected following the recommendations of the Prisma strategy. The results pointed to 3 thematic categories, namely: religiosity as a risk or protection factor in sexuality during the health/illness process, religiosity and sexuality in the life cycle and in population groups, and religiosity and sexuality in the prevention of HIV/AIDS. It was concluded that religion could interfere in the health-disease process; studying it is necessary for the understanding of the individual in different spheres, so that proposals of interventions can be applied according to the needs of the subject.
\end{abstract}

Keywords: Religiosity; Sexuality; Health / disease process; Care; Health.

\section{Resumen}

Se han estudiado a lo largo de los años sobre la influencia de la religión en las prácticas sexuales humanas. El objetivo há sido conocer la producción científica nacional e internacional sobre la relación entre sexualidad y religiosidad de 2012 a 2017. Se trata de una revisión integradora de la literatura. Se utilizaron las bases de datos Scielo, Pubmed y BVS y se encontraron 167 artículos, de los cuales 40 han sido seleccionados siguiendo las recomendaciones de la estrategia PRISMA. Los resultados apuntaron a 3 categorías temáticas, a saber: la religiosidad como factor de riesgo o protección de la sexualidad durante el proceso de salud / enfermedad, la religiosidad y sexualidad en el ciclo de vida y en grupos de población, y la religiosidad y sexualidad en la prevención del VIH / SIDA. Se concluye que la religión 
puede interferir en el proceso salud-enfermedad; estudiarlo es necesario para la comprensión del individuo en diferentes ámbitos, y para que las propuestas de intervenciones puedan ser aplicadas según las necesidades del sujeto.

Palabras clave: Religiosidad; Sexualidad; Proceso de salud/enfermedad; Cuidado; Salud.

\section{Introdução}

A influência da religião na sexualidade tem sido investigada pela literatura internacional por mais de meio século (Coutinho \& Miranda-Ribeiro, 2014). Para uma melhor compreensão, em princípio, destaca-se o conceito de sexualidade problematizado pela Organização Mundial de Saúde (OMS) e o de religiosidade definido por alguns autores.

De acordo com a OMS (2001, p. 8), a sexualidade pode ser entendida como uma força que motiva o indivíduo ir à busca de: contato, calor humano, de sentimentos como: afeto, amor, carinho e ternura; ela está interligada (e interfere diretamente) à maneira como o ser humano sente, se move, toca e é tocado. A sexualidade tem uma forte influência sobre os pensamentos, interações e relações, influenciando, por sua vez, a saúde física e mental do indivíduo.

Gomes et al. (2014) explicam a religiosidade como um conjunto de dogmas e regras institucionais ligados a uma ou mais religiões. Já para Catré et al (2016), esta pode se apresentar enquanto vertente mais privada e individual assim como uma vertente pública, social e institucional, podendo ser classificada como religiosidade "intrínseca" e "extrínseca", intrínseca quando o indivíduo vive a religião e, extrínseca quando se usa a religião para os seus fins próprios.

A religiosidade contribui com a construção subjetiva de que existe um ser ou dimensão maior, que mantém o controle sobre o fluxo da vida e seus acontecimentos, dando ao sujeito capacidade para lidar com as diversas eventualidades (positivas ou negativas) de maneira equilibrada e confiante, diminuindo sentimentos como estresse e ansiedade (Gomes et al., 2014).

É evidente a possível influência da religião na saúde sexual e reprodutiva no que se refere ao conhecimento, atitudes e práticas relacionadas à sexualidade, o que demonstra a necessidade de intensificar as pesquisas com relação às múltiplas variáveis que podem estar associadas à religião (Coutinho \& Miranda-Ribeiro, 2014).

Em algumas das pesquisas mais recentes, encontramos essa associação da religiosidade na sexualidade dos indivíduos. Johnson (2018) relata sobre os enfretamentos de mulheres católicas frente às diferentes negociações entre religiosidade e aborto, e Felitti (2016) analisa o entendimento relacionado ao gênero, à sexualidade, espiritualidade/religiosidade (entre outros aspectos), relativos ao ciclo menstrual na Argentina.

Sendo assim, tem-se como questão norteadora deste estudo: qual a produção científica nacional e internacional acerca da relação entre as práticas sexuais e a religiosidade, entre os anos de 2012 a 2017 ?

\section{Metodologia}

Trata-se de uma revisão integrativa de literatura, que é considerada uma das abordagens mais amplas das revisões, pois pode se utilizar tanto elementos teóricos como empíricos, sendo relevante, na ampla amostra e propostas de estudos, conceitos e problemas de saúde (Souza et al., 2010).

Uma revisão integrativa no geral segue 6 etapas distintas, a saber: a elaboração do tema e a seleção da questão de pesquisa; estabelecimento dos critérios de inclusão e exclusão e busca na literatura; definição das informações a serem extraídas dos estudos selecionados e categorização dos estudos; avaliação dos estudos incluídos na revisão integrativa; interpretação dos resultados; apresentação da revisão e síntese do conhecimento (Mendes et al., 2008).

A busca dos estudos foi realizada no mês de novembro de 2018, por meio das seguintes ferramentas de busca e bases de dados: Biblioteca Virtual em Saúde (BVS), Scientific Electronic Library Online (SCIELO) e PubMed, utilizando os filtros relacionados aos anos de 2012 a 2017.

Os descritores em ciências da saúde (DeCS) utilizados e combinados foram: "sexuality" e "religion". A estratégia de busca adotada foi "sexuality" AND "religion”, pois foi a combinação de descritores que mais apresentou artigos. 
Os critérios de inclusão adotados para a seleção dos artigos foram: estudos escritos na forma de artigo eletrônico, nos idiomas português, inglês e espanhol, que tratam da relação entre sexualidade e religiosidade; artigos originais. Os critérios de exclusão foram: artigos não disponíveis no formato eletrônico; e/ou que não respondessem à pergunta norteadora e artigos de revisão, editoriais e/ou relatos de experiência.

O processo de busca e seleção dos estudos seguiu as recomendações PRISMA e está representado no Quadro 1.

Quadro 1. Fluxograma dos métodos de identificação, triagem, elegibilidade e inclusão dos artigos de acordo com PRISMA Flow Diagram. Santo Antônio de Jesus, Bahia, Brasil, 2018.

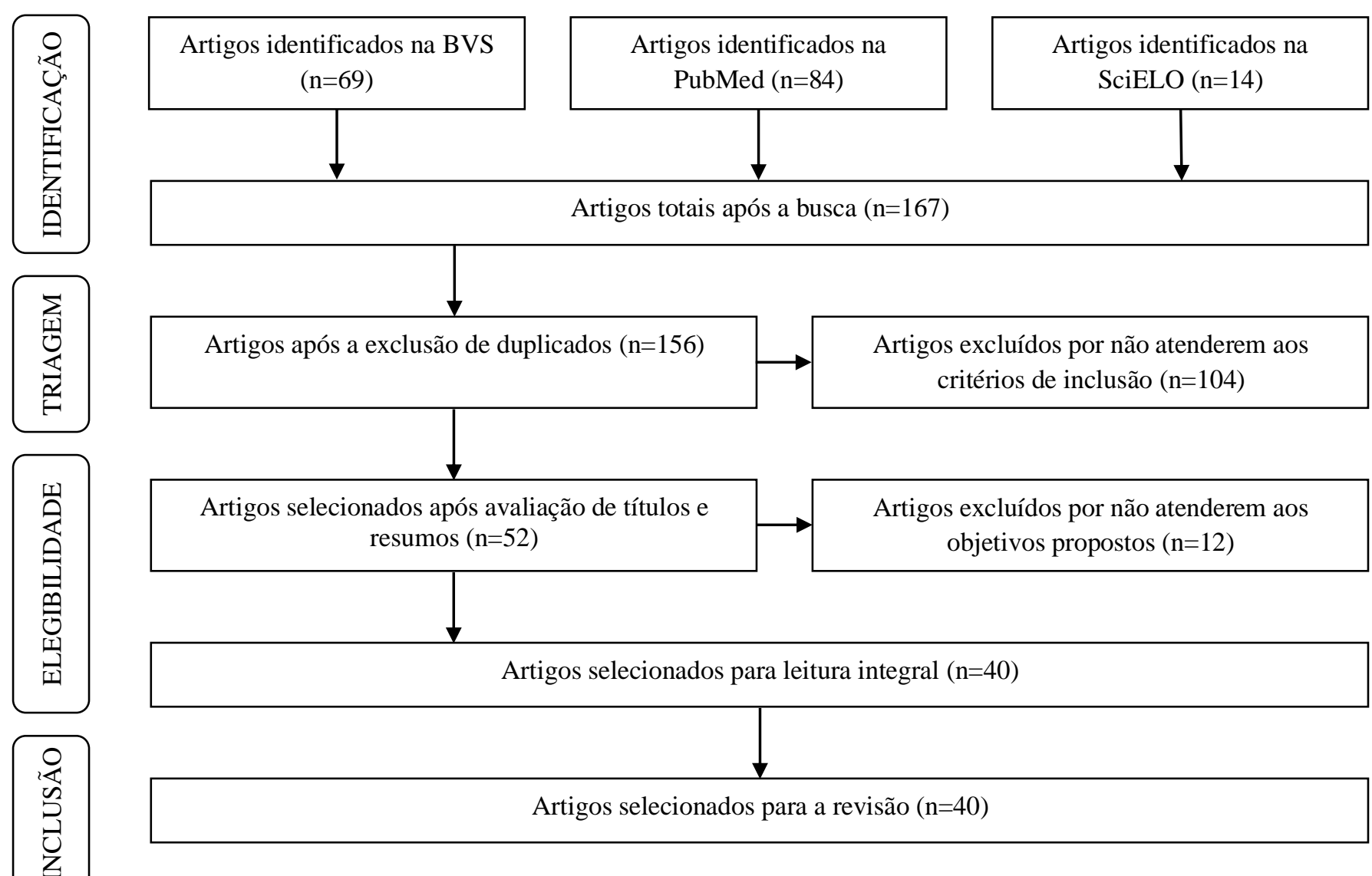

Fonte: PRISMA Group.

Durante a etapa de sistematização dos artigos foi utilizado o software ENDNOTE® para importar as referências e, organizá-las nos seguintes agrupamentos: "válidos" (atendiam aos objetivos da pesquisa), "excluídos" (não atendiam aos objetivos da pesquisa) e "duplicados" (constam tanto na BVS quanto no SCIELO e/ou no PubMed). A sistematização foi feita por três revisoras e, em caso de dúvidas, os artigos foram revistos por uma quarta revisora especialista na área de saúde mental, a fim de sanar as dúvidas e obter o total de artigos válidos.

A análise e a discussão dos resultados obtidos basearam-se no confronto dos achados do presente estudo com artigos da literatura internacional e nacional sobre religiosidade e práticas sexuais.

\section{Resultados}

Foram encontrados 84 artigos no PubMed, 69 artigos na BVS e 14 artigos na Scielo. Após a leitura dos títulos e resumos, tendo como base os critérios de inclusão e exclusão e os objetivos da revisão, foram selecionados 40 artigos para 
serem lidos por completo e agrupados em categorias temáticas convergentes. Após a leitura dos 40 artigos foi possível apreender três categorias temáticas, a saber: a religiosidade como fator de risco ou proteção na sexualidade durante o processo saúde-doença; a religiosidade e sexualidade no ciclo vital e em grupos populacionais e, a religiosidade e a sexualidade na prevenção de HIV/AIDS (Quadro 2). É válido ressaltar que um mesmo artigo pode estar contido em mais de uma categoria.

Quadro 2. Número de artigos (n) presentes em cada categoria temática. Santo Antônio de Jesus, 2018.

\begin{tabular}{|l|c|}
\hline \multicolumn{1}{|c|}{ Categorias Temáticas } & $\begin{array}{c}\text { Número de artigos } \\
\text { (n) }\end{array}$ \\
\hline $\begin{array}{l}\text { Categoria 1 - A religiosidade como fator de risco ou proteção na sexualidade durante } \\
\text { o processo saúde-doença }\end{array}$ & 22 \\
\hline $\begin{array}{l}\text { Categoria 2 - A religiosidade e a sexualidade no ciclo vital e em grupos } \\
\text { populacionais }\end{array}$ & 21 \\
\hline Categoria 3 - A religiosidade e a sexualidade na prevenção de HIV/AIDS & 7 \\
\hline
\end{tabular}

Fonte: Autores.

$\mathrm{Na}$ categoria "a religiosidade como fator de risco ou proteção na sexualidade durante o processo saúde-doença", quanto às abordagens foram encontrados 11 artigos que utilizaram o método quantitativo, 10 que utilizaram o método qualitativo e 1 usou o método quanti-qualitativo. Nessa categoria, os artigos buscaram compreender como a religiosidade, por meio da moralidade, dogmas e ética religiosa, pode influenciar na orientação, comportamento e práticas sexuais. Assim, os estudos, geralmente, têm abordado a relação religiosidade/sexualidade a partir do fator de risco e/ou proteção para o desenvolvimento de comorbidades psiquiátricas, discriminação social, contracepção e homofobia internalizada. As religiões abordadas nos artigos foram, majoritariamente, o judaísmo, as vertentes do cristianismo, ou, simplesmente, foram trazidos os termos "religiosidade" e "espiritualidade", sem a preocupação do aprofundamento teórico. Os cenários dos artigos foram diversos e, perpassaram por países dos continentes africano, europeu, americano, oceânico e asiático.

Foram encontrados 21 artigos relativos à categoria "a religiosidade e a sexualidade no ciclo vital e em grupos populacionais" que versavam sobre como a religiosidade e a sexualidade são vivenciadas por faixas etárias diferentes, gêneros e orientação sexual. Sendo assim, nessa categoria, 11 artigos tiveram como alvo de discussão a faixa etária populacional, ou seja, jovens, adultos e, idosos. 3 artigos buscaram compreender essa relação a partir do recorte de gênero, ou seja, mulheres cisgêneras, homens cisgêneros e, mulheres transgêneras. Já 7 artigos discutem a partir da perspectiva das orientações sexuais: heterossexuais, homossexuais e população de Lésbicas, Gays, Bissexuais, Transgêneros, Queers, Intersexuais, Assexuais e outros (LGBTQIA+). Dos artigos, 13 utilizaram abordagens quantitativas, 7 usaram de abordagens qualitativas e 1 classifica sua abordagem enquanto quanti-qualitativa. Os cenários dos estudos foram os continentes europeu, americano, africano e asiático.

No que tange à categoria temática "A sexualidade e a religiosidade na prevenção de HIV/AIDS", 7 artigos se preocuparam com a saúde sexual, ou seja, estudaram como a religiosidade e a sexualidade podem ser preditivas ou não para a infecção ou prevenção do HIV/AIDS. Os cenários dos estudos foram variados, sendo possível encontrar artigos no contexto de países como as Filipinas, Zimbábue, África do Sul, Nigéria, República do Congo e Dinamarca. Nesses artigos, a religião em relação à saúde sexual tanto é entendida como colaboradora, ao informar sobre contracepção, quanto desmotivadora, ao reprovar o uso de contraceptivos. Assim, além dos cenários diversos, as abordagens utilizadas foram: qualitativas (4 artigos) e quantitativas (3 artigos). O Quadro 3 abaixo descreve os artigos conforme às categorias apresentadas, o tipo de abordagem utilizada, o cenário do estudo e a religião. 
Quadro 3. Sistematização dos artigos conforme as categorias temáticas, o tipo de estudo, o cenário e a religião. Santo Antônio de Jesus, 2019.

\begin{tabular}{|c|c|c|c|c|c|c|}
\hline \multirow{2}{*}{ Autoria } & \multicolumn{3}{|c|}{ Categoria } & \multirow{2}{*}{$\begin{array}{l}\text { Tipo de } \\
\text { Estudo }\end{array}$} & \multirow{2}{*}{ Cenário } & \multirow{2}{*}{ Expressão Religiosa } \\
\hline & 1 & 2 & 3 & & & \\
\hline Hill, Siwatu \& Robinson, 2014 & Sim & Não & Não & Quantitativo & EUA & Catolicismo e Protestantismo \\
\hline Pedersen, 2014 & Não & Sim & Não & Quantitativo & Noruega & Cristianismo \\
\hline Meladze, \& Brown, 2015 & Sim & Sim & Não & Quantitativo & Ásia/Ocidente & $\begin{array}{l}\text { Fé abraâmica, religiões filosóficas e da nova } \\
\text { era }\end{array}$ \\
\hline Korup et al., 2016 & Não & Não & Sim & Quantitativo & Dinamarca & Adventista do $7^{\circ}$ dia e Batista \\
\hline Lytle et al. 2015 & Não & Sim & Não & Quantitativo & EUA & Cristianismo \\
\hline Lusey et al., 2014 & Sim & Não & Sim & Qualitativo & Congo & $\begin{array}{l}\text { Exército da Salvação, Protestantismo, Igreja } \\
\text { do Ressuscitado }\end{array}$ \\
\hline Li et al. 2016 & Sim & Sim & Não & Quantitativo & EUA & Protestantismo \\
\hline Olaore, \& Olaore, 2014 & Sim & Não & Sim & Qualitativo & Nigéria & Cristianismo \\
\hline Osafo, 2014 & Sim & Sim & Não & Qualitativo & Ghana & Cristianismo \\
\hline Munyaradzi Kenneth et al., 2016 & Sim & Sim & Não & Quantitativo & Zimbábue & Seita apostólica \\
\hline Heeren et al., 2014 & Sim & Não & Sim & Quantitativo & África do Sul & Religiosidade \\
\hline Lassiter et al., 2017 & Não & Sim & Não & Quantitativo & EUA & Espiritualidade e religiosidade \\
\hline Iveniuk et al., 2016 & Não & Sim & Não & Quantitativo & EUA & Judaísmo e Cristianismo \\
\hline Manzou et al., 2014 & Não & Não & Sim & Quantitativo & Zimbábue & Cristianismo, Espiritualismo e Tradicionais \\
\hline Norder et al., 2015 & Não & Não & Sim & Qualitativo & África do Sul & $\begin{array}{l}\text { Pentecostal, Apostólica, Anglicana, e Uniting } \\
\text { reformed church }\end{array}$ \\
\hline Lucea et al., 2013 & Sim & Sim & Sim & Qualitativo & Filipinas & Religiosidade \\
\hline Lewczuk et al., 2017 & Sim & Não & Não & Quanti/Quali & Polônia & Religiosidade \\
\hline Cantarino et al., 2016 & Não & Não & Sim & Qualitativo & Espanha & Religiosidade e Espiritualidade \\
\hline David, \& Weitzman, 2015 & Não & Sim & Não & Qualitativo & - & Judaísmo \\
\hline Drabble et al., 2016 & Sim & Não & Não & Qualitativo & EUA & $\begin{array}{l}\text { Religiosidade, Catolicismo, Protestantismo e } \\
\text { Judaísmo }\end{array}$ \\
\hline Garofalo et al., 2014 & Sim & Não & Não & Quantitativo & EUA & Protestantismo, Catolicismo e outras religiões \\
\hline Gattis et al., 2014 & Sim & Não & Não & Quantitativo & EUA & Não especificou \\
\hline Gibbs, \& Goldbach, 2015 & Não & Sim & Não & Quantitativo & - & Cristianismo e outras religiões \\
\hline Gymah et al., 2013 & Não & Sim & Não & Quantitativo & Quênia & $\begin{array}{l}\text { Religiões pentecostais evangélicas e outras } \\
\text { religiões cristãs }\end{array}$ \\
\hline $\begin{array}{l}\text { Pietkiewicz, \& Kolodziejczyk- } \\
\text { Skrzypek, } 2016\end{array}$ & Sim & Não & Não & Qualitativo & Polônia & Catolicismo \\
\hline Quinn, \& Dickson-Gomez, 2016 & Sim & Não & Não & Qualitativo & EUA & Igreja Negra \\
\hline Quinn et al., 2015 & Não & Sim & Não & Qualitativo & EUA & Igreja Negra \\
\hline Sanabria Mazo et al., 2016 & Não & Sim & Não & Qualitativo & Colômbia & Catolicismo \\
\hline Shalev et al., 2013 & Sim & Não & Não & Qualitativo & Israel & Igreja Ortodoxa \\
\hline Silva et al., 2008 & Sim & Não & Não & Qualitativo & Brasil & $\begin{array}{l}\text { Catolicismo, Umbanda, Candomblé e } \\
\text { Evangélicas de diferentes denominações }\end{array}$ \\
\hline Smallwood et al., 2016 & Sim & Não & Não & Quantitativo & EUA & Igreja Negra \\
\hline Sowe et al, 2016 & Sim & Não & Não & Quantitativo & Austrália & Cristianismo \\
\hline Sümer, 2015 & Sim & Não & Não & Quantitativo & Turquia & Não especificado \\
\hline Williams et al., 2014 & Não & Sim & Não & Qualitativo & EUA & Igreja Cristã Afro-americana \\
\hline Rios et al., 2008 & Sim & Não & Não & Qualitativo & Brasil & Catolicismo e Protestantismo \\
\hline Van Drie et al., 2014 & Não & Sim & Não & Quanti/Quali & Holanda & Protestantismo \\
\hline Winter et al, 2013 & Sim & Sim & Não & Quantitativo & Finlândia & Igreja Lutérica Evangélica finlandesa \\
\hline Yasmine et al., 2015 & Não & Sim & Não & Quantitativo & Líbano & Não especificado \\
\hline Zarzycka et al., 2017 & Não & Sim & Não & Quantitativo & Polônia & Catolicismo \\
\hline Lejeune, 2013 & Não & Sim & Não & Quantitativo & EUA & Não especificado \\
\hline
\end{tabular}

Fonte: Autores.

\section{Discussão}

A religiosidade pode ser entendida como uma via importante para a promoção da saúde, sobretudo, a sexual. Com base nisso, 22 artigos desta revisão entendem que a religião, por meio da moralidade e dos preceitos, pode atuar como um fator de proteção ao estimular a abstinência sexual, o sexo depois do casamento e números menores de parceiros sexuais (Hill et al., 2014; Li, Freedman et al., 2016; Olaore \& Olaore, 2014, Munyaradzi Kenneth et al., 2016; Drabble et al., 2016; Garofalo et al., 2014; van Drie et al., 2014). 
Assim, para outros autores a ética religiosa pode auxiliar num comportamento sexual mais seguro, regulatório e protetivo em relação a algumas doenças, como as Infecções Sexualmente Transmissíveis (ISTs) (Olaore \& Olaore, 2014). Em um estudo com adolescentes universitários, verificou-se que ter uma afiliação religiosa contribuía para que os jovens se responsabilizassem em relação à prática sexual, considerando-se que, desta forma, os praticantes de tais religiões contavam, anteriormente à iniciação da vida sexual, com uma maior maturidade espiritual (Sanabria Mazo et al., 2016).

Outro estudo destacou o papel dos líderes religiosos, pois muitas vezes são os que têm mais contato com os jovens, que podem orientá-los em relação ao uso de contraceptivos e quebrar alguns tabus relacionados à sexualidade e aos relacionamentos amorosos ( $\mathrm{Li}$ et al., 2016). No entanto, a sexualidade ainda é tratada como tabu em muitas comunidades religiosas. Noutro estudo realizado no Congo, muitos dos entrevistados disseram que não se deve falar sobre sexo ou sexualidade no ambiente da Igreja, pois esse tema é de foro íntimo. É válido ressaltar que, no Congo, há uma incidência alta de HIV/ AIDS e o uso de preservativos é, muitas vezes, desaconselhado por alguns líderes religiosos (Olaore \& Olaore, 2014). Nesse sentido, a religião pode também ser um fator de risco no processo saúde-doença, sobretudo no que se refere à disseminação de algumas IST's, dentre elas, o HIV/ AIDS (Lusey et al, 2014; Olaore \& Olaore, 2014).

Em uma pesquisa realizada com 84 jovens católicas, foi possível apreender que os discursos em relação à prevenção das ISTs, por vezes se esgotam na questão da fidelidade e do sexo após o casamento, havendo uma culpabilização individual e o aumento da vulnerabilidade às ISTs (Couto et al, 2017). Essa moralidade religiosa também pode ser um fator de risco ao propagar discursos que não contemplam as diversas orientações sexuais e as múltiplas identidades de gênero, servindo de base para discriminação e violências como a homofobia internalizada (Gibbs \& Goldbach, 2015; Pietkjewica \& KolodziejczykSkrzypek, 2016).

Diante de um discurso ainda vigente, de que a sexualidade está atrelada à procriação biológica e à perpetuação exclusiva de um modelo heteronormativo de família, há na contemporaneidade, uma "guerra" sexual e cultural que envolve os preceitos religiosos e suas dificuldades de abarcar as liberdades sexuais, as identidades de gênero e as orientações sexuais diversas (Natividade \& Oliveira, 2013). Essa tensão é, muitas das vezes, causadora de experiências de homonegatividade, em que muitas pessoas precisam esconder sua expressão sexual diante de familiares e da comunidade da igreja, para evitar situações de insultos, alienação e exclusão social, fazendo com que esse grupo tenha maior probabilidade de desenvolver pensamentos suicidas crônicos e um sentimento de maior aflição ao lidar com uma sexualidade proibida pela religião (Gibbs \& Goldbach, 2015, Quinn et al., 2016).

Na contramão da exclusão social, tem crescido no Brasil o movimento de igrejas protestantes chamadas de "igrejas inclusivas", que utilizam a persuasão e discursos para promover a inclusão da comunidade LGBTQIA+ em seus espaços de oração e fé. Essas agremiações religiosas sofrem pressão das igrejas mais tradicionais, porém têm ampliado seu público e incluído pessoas com sexualidades e identidades de gênero que fogem da heteronormatividade (Silva, 2018).

Sendo assim, neste estudo além de a religiosidade figurar como fator de risco ou de proteção no processo saúdedoença, a abordagem central de alguns artigos trouxe, também, a interlocução entre religiosidade e sexualidade a partir de vivências dos indivíduos em determinadas faixas etárias, gênero ou grupos populacionais específicos, como por exemplo, mulheres transgêneras e idosas.

Desta forma, a segunda categoria "religiosidade e sexualidade no ciclo vital e em grupos populacionais" emergiu a partir da forma como a experiência religiosa tem se relacionado com as práticas sexuais, expressões de sexualidade, ou com questões de saúde sexual. Entre jovens de 18 a 30 anos, a religião pode ser um fator de influência quanto à idade da primeira relação sexual, se ocorre antes ou após a união matrimonial, e a maneira como lidam com métodos contraceptivos e a incidência destas.

Dentre os jovens que indicaram nunca terem praticado sexo oral, anal ou vaginal $(\mathrm{n}=895), 70 \%(\mathrm{n}=626,5)$ 
justificaram que suas preocupações eram advindas de concepções religiosas, enquanto que 58\% (n=519,1) temiam rejeição social, e 61\% (n=545,95) a desaprovação dos pais (Yasmine et al., 2015). O sistema religioso voltado para o enfoque do sexo como pecado, enfatiza que se não estiverem associados a ele o casamento, a monogamia e a procriação, então a prática sexual é tida como ilegítima e sem virtudes (Couto, 2016). Isto é, infere-se que a determinação religiosa e seu incentivo para que as relações sexuais aconteçam apenas em contexto conjugal, muitas vezes confrontado com os valores culturais atuais, pode desencadear implicações de ordem emocional.

Couto et al. (2017), em outro estudo, e em concordância com o que pode ser observado nos achados desta revisão, revelou o consenso entre jovens católicos quanto às dúvidas sobre práticas de "sexo seguro", e à necessidade da aproximação dos discursos religioso e científico. Afinal, de acordo com os resultados encontrados, a percepção de alguns pais de duas comunidades ganesas quanto à influência religiosa no comportamento sexual de seus filhos é a de que, associada ao diálogo, ela executa um papel protetivo, restringindo os adolescentes de um comportamento sexual de risco (Osafo, 2014).

Quando analisados a partir da ótica da identidade sexual ou de gênero, a religiosidade aparece relacionada a termos como homonegatividade, LGBTQIA+fobia internalizada, e como precursora de pensamentos suicidas. A orientação sexual, por exemplo, foi decisiva em aspectos como desconforto religioso, ansiedade e insatisfação com a vida, uma vez que os maiores índices de interações religiosas negativas, como desaprovações críticas, foram observados em participantes homossexuais (Zarzycka et al., 2017).

Segundo Silva (2018), isso ocorre como consequência da presença de elementos excludentes ao grupo LGBTQIA+ nos espaços ditos transcendentais, como a fabricação de discursos preconceituosos direcionados a esse grupo, e a alimentação desses discursos dirigidos à toda a sociedade, na tentativa de legitimar a superioridade dos "normais" sobre esse grupo "pecador". Apesar disso, algumas pessoas que se identificam como LGBTQIA+, conseguem conciliar sua afiliação religiosa com a sua sexualidade, seja por meio de maior identificação com a espiritualidade ou com as ditas religiões aliadas.

Estudantes LGBTQIA+ apresentaram maiores índices de ideação suicida ativa do que aqueles não-LGBTQIA+ quando comparados apenas dentro de seu grupo religioso (LGBTQIA+ cristãos versus não-LGBTQIA+ também cristãos), porém, estudantes LGBTQIA+ ateus/agnósticos apresentaram maiores índices de "desejo de morte" do que os jovens LGBTQIA+ cristãos e judeus (Lytle et al., 2015). Infere-se, então, que a religiosidade pode configurar um fator importante na promoção da saúde mental das pessoas LGBTQIA+ quando se relaciona a um ambiente de interação social acolhedor e contribui para a interação e formação de rede de apoio.

A igreja pode ter grande importância aos seus frequentadores para além do estudo religioso e suas implicações. Ela tem participação na construção identitária e nos vínculos sociais de seus integrantes, bem como muitas vezes desempenha um papel protetivo a determinados grupos ali existentes. É o caso da Black Church, nos Estados Unidos. Apesar da prevalência da homonegatividade dentro da igreja, homens negros bissexuais e gays seguem frequentando-as, uma vez que consideram importante seu envolvimento de integração familiar e comunitária (Quinn et al., 2016). É como Silva et al. (2017) trazem em seus estudos realizados no Brasil, a respeito das igrejas cristãs. Eles apontam que a maioria dessas igrejas não rompem com a tradição dogmática repressora quando o tema é a moral sexual. Apesar disso, através do pluralismo religioso, ocorre o surgimento de novas experiências de fé e vertentes que buscam compatibilidade entre o cristianismo e sexualidades não heterossexuais e/ou a transgeneridade.

Em alguns casos, inclusive, grupos religiosos podem surgir a partir de demandas específicas dos praticantes de determinada religião e unirem-se ao sistema de saúde local na promoção da saúde ou na prevenção de doenças. São os casos da Igreja Apostólica, que oferece um serviço conjunto voltado para a saúde da mulher, e a Igreja Católica, com um serviço voltado para jovens quanto à prevenção de ISTs (Munyaradzi Kenneth et al., 2016; Lucea et al, 2013). Apesar de o apostolicismo promover o casamento precoce, a fertilidade e o não uso de métodos contraceptivos, a existência de um grupo 
dessa afiliação religiosa no Zimbábue, voltado para o suporte à saúde da mulher, demonstra o desejo dessa comunidade em reverter quadros de demora no reconhecimento de sinais de perigo da exposição a práticas sexuais não seguras. Bem como faz a Igreja Católica em parceria com o sistema de saúde das Filipinas por meio de programas de intervenção focados na desestigmatização e no incentivo ao uso de camisinha no combate às ISTs. Principalmente o HIV/AIDS, tema bastante abordado nos achados da presente pesquisa, dando origem à terceira categoria dos resultados: a religiosidade e a sexualidade na prevenção de HIV/AIDS.

Conforme Pinho et al. (2017), a AIDS é uma doença crônica, e seu diagnóstico impacta na vida do indivíduo de tal modo que pode provocar alterações nas esferas psicológicas, fisiológicas e sociais. Porém, se devidamente tratada, tem a probabilidade de redução da transmissão do vírus, do adoecimento físico e mental, mortalidade, além da melhoria na qualidade de vida da Pessoa Vivendo com HIV/AIDS (PVHA). Pinho et al. (2017) afirmam que tal melhoria pode ser alcançada por meio do Copyng Religioso-Espiritual (CRE), sendo este um conjunto de estratégias cognitivas e comportamentais para o enfrentamento de situações de estresse ou risco, de acordo com Helena \& Polakiewicz (2015), que é de extrema importância para as PVHA no enfrentamento positivo da doença.

Corroborando com esses autores, a religiosidade associada a momentos de discussão sobre o uso de preservativos e métodos contraceptivos, serviu como atitude de fator protetivo em relação ao sexo com parceiros fixos e casuais (Heeren et al., 2014). Ressaltando a importância da discussão do tema dentro do ambiente religioso, Couto et al. (2017) afirmam que a falta de informação ou informações superficiais e/ou contraditórias acerca da sexualidade e da prática do sexo seguro, podem ser fatores que potencializam a vulnerabilidade de jovens religiosos às ISTs, destacando-se o HIV, assim como a dogmatização da temática que estão no campo do conhecimento científico.

Infere-se que a religiosidade serve de fator protetivo ao HIV/AIDS por incentivar um estilo de vida relativamente mais tranquilo, que reforça comportamentos que não oferecem riscos à saúde dos adeptos a tal prática religiosa. Assim, o comportamento sexual de risco é diminuído, o que poderia explicar a menor incidência de ISTs. Santos et al. (2014) revelaram a relação da religiosidade e das práticas corporais como um meio de apoio para a adoção de um estilo de vida ativo, como a prática de exercício físico e hábitos alimentares saudáveis. Assim como a relação da religiosidade e substâncias psicoativas, algumas religiões influenciam no menor ou nenhum consumo de álcool e outras drogas, afetando positivamente a vida dos religiosos em relação a comportamentos de risco, como relações sem proteção, por exemplo, que poderiam, por sua vez, expôlos ao HIV/AIDS (Manzou et al, 2014).

Dos artigos encontrados nesta categoria, 70\% foram realizados no continente africano, sendo apenas um artigo do continente europeu e outro asiático. Isso reforça que há um enorme estigma na relação África - HIV/AIDS que, segundo Sanabria Mazo (2016, p.27), é decorrente principalmente na parte Sul da África, por questões ligadas a um "caráter patogênico geopolítico", e que também é reforçado por uma série de produções científicas que interligam comportamento sexual, raça e nação, e tantas outras produções a respeito do surgimento do HIV/AIDS, tendo o continente como "berço".

\section{Considerações Finais}

A religião tem um papel importante em diversos aspectos da vida dos indivíduos que a praticam, inclusive na vivência da sexualidade. Ela perpassa gerações e, muitas vezes, pode ser compreendida como uma via importante tanto para a promoção como um fator de risco à saúde.

Por meio da moralidade e dos preceitos, a religião pode atuar como um fator de proteção ao estimular a abstinência sexual, o sexo depois do casamento e números menores de parceiros sexuais. No entanto, a sexualidade como um todo ainda é tratada como tabu em muitas comunidades religiosas; onde o uso de preservativos é muitas vezes desaconselhado e deve-se evitar falar sobre sexo ou sexualidade, por ser considerado um tema de foro íntimo. Nesse sentido, a religião pode se 
configurar como um fator de risco no processo saúde/doença.

Nota-se, que a experienciação da sexualidade carrega significados e contextos diferentes a depender da faixa etária e do grupo populacional. Assim, no ciclo vital, muitas religiões são atuantes no aconselhamento de jovens em iniciação sexual e também nas questões como companheirismo e sexo entre a população idosa. Além do impacto da religião no ciclo vital, em grupos populacionais, como a população LGBTQIA+, os dogmas podem ser responsáveis pelo desenvolvimento da homofobia internalizada e pelo sofrimento psíquico de pessoas que praticam a religião, mas possuem orientações sexuais e identidades de gênero que não são reconhecidas pelos grupos religiosos. No entanto, ficou evidente nos estudos, que sentir-se parte de uma comunidade religiosa é importante para os mais diversos grupos de pessoas, mesmo quando esse espaço da religião não abarca as orientações sexuais e identidades de gênero dos sujeitos.

Como os cenários desta revisão contemplaram países e continentes diversos, observou-se que muitos estudos do continente africano se preocupavam em conhecer como essa relação sexualidade/religiosidade pode atuar na prevenção e redução de HIV/AIDS. Nesse sentido, pode-se apreender que a depender da conscientização de líderes religiosos, a religião pode ser uma aliada à propagação de formas de prevenção a ISTs e da saúde sexual. No entanto, ainda uma grande barreira se estabelece quando os discursos religiosos não positivam uso de contraceptivos e prevenção, sendo, por vezes, maior a disseminação de doenças sexualmente transmissíveis.

Diante do exposto, faz-se necessário estudar as religiosidades visando trabalhar o tema da sexualidade tanto nos serviços quanto nos cursos de saúde para compreender o indivíduo a partir das diversas esferas: biológicas, espirituais, sociais; para que seja possível que se proponha uma intervenção diferenciada e benéfica, de acordo com as necessidades do sujeito.

\section{Referências}

Camboim, A., \& Rique, J. (2010). Religiosidade e espiritualidade de adolescentes e jovens adultos. Rev Brasil Hist Relig., 3(7), 251-263. https://doi.org/10.4025/rbhranpuh.v3i7.30336.

Cantarino, S. G., Pinto, J. M. M, Fabião, J. A. S. A. O., García, A. M. C., Abellán, M. V., \& M Rodrigues, M. A. (2016). The Importance of Religiosity/Spirituality in the Sexuality of Pregnant and Postpartum Women. PLOS ONE, 11(6). https://doi.org/10.1371/journal.pone.0156809

Catré, M. N. C., Ferreira, J. A., Pessoa, T., Catré, A., \& Catré, M. C. (2016). Espiritualidade: Contributos para uma clarificação dos conceitos. Análise Psicológica, 34(1), 31-46. https://dx.doi.org/10.14417/ap.877

Coutinho, R. Z., \& Miranda-Ribeiro, P. (2014). Religião, religiosidade e iniciação sexual na adolescência e juventude: lições de uma revisão bibliográfica sistemática de mais de meio século de pesquisas. Revista Brasileira de Estudos de População, 31(2), 333-365. https://doi.org/10.1590/S010230982014000200006

Couto, P. L. S., Paiva M. S., Gomes, A. M. T., Boa Sorte, E. T., Rodrigues, L. S. A., \& Coelho, E. A. (2017). Significados a respeito da prevenção ao HIV/aids e da sexualidade para jovens católicos. Rev Gaúcha Enferm., 38(4). https://www.scielo.br/pdf/rgenf/v38n4/1983-1447-rgenf-38-04-e2016-0080.pdf

Couto, P. L. S. (2016). A influência da religião católica entre os/as jovens sobre o exercício da sexualidade e a prevenção do HIV/AIDS. [Dis sertação de Mestrado, Escola de Enfermagem - Universidade Federal da Bahia]. https://repositorio.ufba.br/ri/handle/ri/18652

David, B. E., \& Weitzman, G. A. (2015). Sexuality in advanced age in Jewish thought and law. J Sex Marital Ther, 41(1), 39-48. https://www.ncbi.nlm.nih.gov/pubmed/24313599

Drabble, L., Trocki, K. F., \& Klinger, J. L. (2016). Religiosity as a protective factor for hazardous drinking and drug use among sexual minority and heterosexual women: Findings from the National Alcohol Survey. Drug and Alcohol Dependence, 161, 127-134. https://doi.org/10.1016/j.drugalcdep.2016.01.022

Felitti, K. (2016). El ciclo menstrual en el siglo XXI. Entre el mercado, la ecología y el poder femenino. Sexualidad, Salud y Sociedad (Rio de Janeiro), (22), 175-208. https://doi.org/10.1590/1984-6487.sess.2016.22.08.a

Garofalo, R., Kuhns, L. M., Hidalgo, M., Gayles, T., Kwon, S., Muldoon, A. L., \& Mustanski, B. (2015). Impact of Religiosity on the Sexual Risk Behaviors of Young Men Who Have Sex With Men. Journal of sex research, 52(5), 590-598. https://doi.org/10.1080/00224499.2014.910290

Gattis, M. N., Woodford, M. R., \& Han, Y. (2014). Discrimination and depressive symptoms among sexual minority youth: is gay-affirming religious affiliation a protective factor?. Archives of sexual behavior, 43(8), 1589-1599. https://doi.org/10.1007/s10508-014-0342-y

Gibbs, J. J., \& Goldbach, J. (2015). Religious Conflict, Sexual Identity, and Suicidal Behaviors among LGBT Young Adults. Arch Suicide Res., 19(4), 472488. https://doi.org/10.1080/13811118.2015.1004476 
Gomes, N., Farina, M., \& Forno, C. (2014). Spirituality, Religion and Religion: Concepts Reflection in Psychological Articles. Revista de Psicologia da IMED, 6(2), 107-112. https://doi.org/10.18256/2175-5027/psico-imed.v6n2p107-112

Gyimah, S., Kodzi, I., Emina, J., Cofie, N., \& Ezeh, A. (2013). Religion, religiosity and premarital sexual attitudes of young people in the informal settlements of Nairobi, Kenya. Journal of Biosocial Science, 45(1), 13-29. https://doi.org/10.1017/S0021932012000168

Heeren, G. A., Icard, L. D., O'Leary, A., Jemmott, J. B., 3rd, Ngwane, Z., \& Mtose, X. (2014). Protective factors and HIV risk behavior among South African men. AIDS and behavior, 18(10), 1991-1997. https://doi.org/10.1007/s10461-014-0767-2

Helena, L. S. M., \& Polakiewicz, R. R. (2015). Coping religioso-espiritual e profissionais da saúde que atuam na área de saúde mental e psiquiatria. Biológicas \& Saúde, 5(18). https://doi.org/10.25242/88685182015771

Hill, N. J., Siwatu, M., \& Robinson, A. K. (2014). "My Religion Picked My Birth Control”: The Influence of Religion on Contraceptive Use. J Relig Health, 53, 825־-833. https://doi.org/10.1007/s10943-013-9678-1

Iveniuk, J., O'Muircheartaigh, C., \& Cagney, K. A. (2016). Religious Influence on Older Americans' Sexual Lives: A Nationally-Representative Profile. Archives of sexual behavior, 45(1), 121-131. https://doi.org/10.1007/s10508-015-0534-0

Johnson, M. C. (2018). "Ni la pareja, ni la familia, ni la Iglesia deciden por mí": la experiencia del aborto en mujeres Católicas. Sexualidad, Salud y Sociedad (Rio de Janeiro), (28), 51-70. https://doi.org/10.1590/1984-6487.sess.2018.28.04.a

Kørup, A. K., Thygesen, L. C., Christensen, R. D. P., Johansen, C., Søndergaard, J., \& Hvidt, N. C. (2016) Association between sexually transmitted disease and church membership a retrospective cohort study of two Danish religious minorities. B M J Open, 6(3). https://doi.org/10.1136/bmjopen-2015-010128

Lassiter, J. M., Saleh, L., Starks, T., Grov, C., Ventuneac, A., \& Parsons, J. T. (2017). Race, ethnicity, religious affiliat ion, and education are associated with gay and bisexual men's religious and spiritual participation and beliefs: Results from the One Thousand Strong cohort. Cultural Diversity and Ethnic Minority Psychology, 23(4), 468-476. https://doi.org/10.1037/cdp0000143

LeJeune, B. C., Zimet, G. D., Azzouz, F., Fortenberry, J. D., \& Aalsma, M. C. (2013). Religiosity and sexual involvement within adolescent romantic couples. Journal of religion and health, 52(3), 804-816. https://doi.org/10.1007/s10943-011-9512-6

Lewczuk, K., Szmyd, J., Skorko, M., \& Gola, M. (2017). Treatment seeking for problematic pornography use among women. Journal of behavioral addictions, 6(4), 445-456. https://doi.org/10.1556/2006.6.2017.063

Li, E., Freedman, L. R., Fernandez y Garcia, E., \& Miller, E. (2016). Exploring the Role of Faith-Based Organizations in Addressing Adolescent Relationship Abuse. Violence Against Women, 22(5), 609-624. https://doi.org/10.1177/1077801215608702

Lucea, M. B., Hindin, M. J., Gultiano, S., Kub, J., \& Rose, L. (2013). The context of condom use among young adults in the Philippines: implications for HIV prevention. Health care for women international, 34(3-4), 227-248. https://doi.org/10.1080/07399332.2012.721414

Lusey, H., San Sebastian, M., Christianson, M., Dahlgren, L., \& Edin, K. E. (2014). Conflicting discourses of church youths on masculinity and sexuality in the context of HIV in Kinshasa, Democratic Republic of Congo. SAHARA-J: Journal of Social Aspects of HIV/AIDS, 11(1), 84-93. https://doi.org/10.1080/17290376.2014.930695

Lytle, M. C., De Luca, S. M., Blosnich, J. R., \& Brownson, C. (2015). Associations of racial/ethnic identities and religious affiliation with suicidal ideation among lesbian, gay, bisexual, and questioning individuals. Journal of affective disorders, 178, 39-45. https://doi.org/10.1016/j.jad.2014.07.039

Manzou, R., Schumacher, C., \& Gregson, S. (2014). Temporal dynamics of religion as a determinant of HIV infection in East Zimbabwe: a serial crosssectional analysis. PLOS ONE, 9(1). https://doi.org/10.1371/journal.pone.0086060

Meladze, P., \& Brown, J. (2015). Religion, Sexuality, and Internalized Homonegativity: Confronting Cognitive Dissonance in the Abrahamic Religions. Journal of religion and health, 54(5), 1950-1962. https://doi.org/10.1007/s10943-015-0018-5

Mendes, K. D. S., Silveira, R. C. C. P., \& Galvão, C. M. (2008). Revisão integrativa: método de pesquisa para a incorporação de evidências na saúde e na enfermagem. Texto \& Contexto - Enfermagem, 17(4), 758-764. https://doi.org/10.1590/S0104-07072008000400018

Munyaradzi Kenneth, D., Marvellous, M., Stanzia, M., \& Memory DM. (2016) Praying until Death: Apostolicism, Delays and Maternal Mortality in Zimbabwe. PLOS ONE, 11(8). https://doi.org/10.1371/journal.pone.0160170

Murakami, R., \& Campos, C. J. G. (2012). Religião e saúde mental: desafio de integrar a religiosidade ao cuidado com o paciente. Revista Brasileira de Enfermagem, 65(2), 361-367. https://doi.org/10.1590/S0034-71672012000200024

Natividade, M., \& Oliveira, L. de. (2013). As novas guerras sexuais: diferença, poder religioso e identidades LGBT no Brasil. Rio de Janeiro: Garamond.

Norder, W. A. J., Peters, R. P. H., Kok, M. O., Elsland, S. L. V., Struthers, H. E., Tutu, M. A., \& Furth, A. M. V. (2015). The church and paediatric HIV care in rural South Africa: a qualitative study. AIDS Care, 27(11), 1404-1409. https://doi.org/10.1080/09540121.2015.1114987

Olaore, I. B., \& Olaore, A. Y. (2014). Is HIV/AIDS a consequence or divine judgment? Implications for faith-based social services. A Nigerian faith-based university's study. SAHARA J: journal of Social Aspects of HIV/AIDS Research Alliance, 11(1), 20-25. https://doi.org/10.1080/17290376.2014.910134

Osafo, J., Asampong, E., Langmagne, S., \& Ahiedeke, C. (2014). Perceptions of parents on how religion influences adolescents' sexual behaviours in two Ghanaian communities: implications for HIV and AIDS prevention. Journal of religion and health, 53(4), 959-971. https://doi.org/10.1007/s10943-013-9689y

Pedersen, W. (2014). Forbidden fruit? A longitudinal study of Christianity, sex, and marriage. Journal of sex research, 51(5), 542-550. https://doi.org/10.1080/00224499.2012.753983 
Pietkiewicz, I. J., \& Kołodziejczyk-Skrzypek, M. (2016). Living in Sin? How Gay Catholics Manage Their Conflicting Sexual and Religious Identities. Archives of sexual behavior, 45(6), 1573-1585. https://doi.org/10.1007/s10508-016-0752-0

Pinho, C. M., Dâmaso, B. F. R., Gomes, E. T., Trajano, M. F. C., Andrade, M. S., \& Valença, M. P. (2017). Religious and spiritual coping in people living with HIV/Aids. Revista Brasileira de Enfermagem, 70(2), 392-399. https://doi.org/10.1590/0034-7167-2015-0170

Moher, D., Liberati, A., Tetzlaff, J., Altman, D. G., \& The PRISMA Group. (2009). Preferred Reporting Items for Systematic Reviews and Meta-Analyses: The PRISMA Statement. PLOS Medicine, 6(7). https://doi.org/10.1371/journal.pmed.1000097

Quinn, K., \& Dickson-Gomez, J. (2016). Homonegativity, Religiosity, and the Intersecting Identities of Young Black Men Who Have Sex with Men. AIDS and behavior, 20(1), 51-64. https://doi.org/10.1007/s10461-015-1200-1

Quinn, K., Dickson-Gomez, J., \& Kelly, J. A. (2016). The role of the Black Church in the lives of young Black men who have sex with men. Culture, health \& sexuality, 18(5), 524-537. https://doi.org/10.1080/13691058.2015.1091509

Rios, L. F., Paiva, V., Maksud, I., Oliveira, C., Cruz, C. M. da S., Silva, C. G. da., Terto Junior, V., \& Parker, R. (2008). Os cuidados com a "carne" na socialização sexual dos jovens. Psicologia em Estudo, 13(4), 673-682. https://dx.doi.org/10.1590/S1413-73722008000400005

Sanabria, Mazo, J. P., Jiménez Sierra, J., Parra Blandón, K., \& Tordecilla Casallas, M. A. (2016). Influencia de la religión en la toma de decisiones sobre sexualidad en estudiantes católicos practicantes. Diversitas, 12(2), 231-242. https://revistas.usantotomas.edu.co/index.php/diversitas/article/view/3246

Sanabria, G. V. (2016). Science, stigmatisation and afro-pessimism in the South African debate on AIDS. Vibrant: Virtual Brazilian Anthropology, 13(1), 2251. https://dx.doi.org/10.1590/1809-43412016v13n1p022

Santos, A. R. M. dos., Silva, E. A. P. C. da., Silva, P. P. C. da., Cartaxo, H. G. de O., \& Freitas, C. M. S. M. de. (2014). Estilo de vida na adolescência: o envolvimento religioso atuando nos comportamentos de risco à saúde. Pensar a Prática, 17(1). https://doi.org/10.5216/rpp.v17i1.18741

Saúde, O. M. (2001). Sexual Health. https://www.who.int/topics/sexual_health/en/

Shalev, O., Baum, N., \& Itzhaki, H. (2013). "There's a man in my bed": the first experience of sex among Modern-Orthodox newlyweds in Israel. Journal of sex \& marital therapy, 39(1), 40-55. https://doi.org/10.1080/0092623X.2012.675021

Silva, B. A. (2018). O discurso da inclusão: Uma análise argumentativa do discurso religioso de uma igreja inclusiva. Tabuleiro das letras, 12(1), 46-60. http://www.revistas.uneb.br/index.php/tabuleirodeletras/article/view/4454

Silva, C. G. da., Santos, A. O., Licciardi, D. C., \& Paiva, V. (2008). Religiosidade, juventude e sexualidade: entre a autonomia e a rigidez. Psicologia em Estudo, 13(4), 683-692. https://doi.org/10.1590/S1413-73722008000400006

Silva, J. B., Simião, J.H., \& Silva, M. R. (2017). Entre o mercado religioso e a fé inclusiva: igrejas LGBT como nicho de mercado. [Cátedra Unesco de Comunicação e Desenvolvimento, Universidade Metodista de São Paulo. XII Conferência Brasileira de Comunicação Eclesial, Campinas: PUC - Campinas], 1-16. https://portal.metodista.br/eclesiocom/2017/arquivos/JefersonBatistadaSILVA_JhonatasHenriqueSIMIO_MarianaRodriguesdaSILVA.pdf

Silva, L.V., \& Barbosa, B. R. S. N. (2016). Sobrevivência no Armário: Dores do Silêncio LGBT em Uma Sociedade de Religiosidade Heteronormativa. Estudos de Religião, 30(3), 129-154. https://doi.org/10.15603/2176-1078/er.v30n3p129-154

Smallwood, S. W., Spencer, S. M., Ingram, L. A., Thrasher, J. F., \& Thompson-Robinson, M. V. (2017). Examining the Relationships Between Religiosity, Spirituality, Internalized Homonegativity, and Condom Use Among African American Men Who Have Sex With Men in the Deep South. American journal of men's health, 11(2), 196-207. https://doi.org/10.1177/1557988315590835

Souza, A. A. de. (2017). O Papel social das Igrejas Evangélicas nas comunidades locais brasileiras. O caso do Projeto Vida Plena na Cidade de Seropédica, Estado do Rio de Janeiro. [Dissertação de Mestrado, Instituto Superior de Ciências Sociais e Políticas]. http://hdl.handle.net/10400.5/14950

Souza, M. T., Silva, M. D., \& Carvalho, R. (2010). Revisão integrativa: o que é e como fazer. Einstein (São Paulo), 8(1), 102-106. https://doi.org/10.1590/s1679-45082010rw1134

Sowe, B. J., Brown, J., \& Taylor, A. J. (2014). Sex and the sinner: comparing religious and nonreligious same-sex attracted adults on internalized homonegativity and distress. The American journal of orthopsychiatry, 84(5), 530-544. https://doi.org/10.1037/ort0000021

Sümer, Z. H. (2015). Gender, Religiosity, Sexual Activity, Sexual Knowledge, and Attitudes Toward Controversial Aspects of Sexuality. Journal of religion and health, 54(6), 2033-2044. https://doi.org/10.1007/s10943-014-9831-5

Van Drie, A., Ganzevoort, R. R., \& Spiering, M. (2014). Anonymous pastoral care for problems pertaining to sexuality. Journal of religion and health, 53(6), 1634-1652. https://doi.org/10.1007/s10943-013-9746-6

Williams, T. T., Dodd, D., Campbell, B., Pichon, L. C., \& Griffith, D. M. (2014). Discussing adolescent sexual health in African-American churches. Journal of religion and health, 53(2), 339-351. https://doi.org/10.1007/s10943-012-9632-7

Winter, T., Karvonen, S., \& Rose, R. J. (2014). Associations between sexual abstinence ideals, religiosity, and alcohol abstinence: a longitudinal study of Finnish twins. Journal of sex research, 51(2), 197-207. https://doi.org/10.1080/00224499.2012.721098

Yasmine, R., El Salibi, N., El Kak, F., \& Ghandour, L. (2015). Postponing sexual debut among university youth: how do men and women differ in their perceptions, values and non-penetrative sexual practices?. Culture, health \& sexuality, 17(5), 555-575. https://doi.org/10.1080/13691058.2014.972457

Zarzycka, B., Rybarski, R., \& Sliwak, J. (2017). The Relationship of Religious Comfort and Struggle with Anxiety and Satisfaction with Life in Roman Catholic Polish Men: The Moderating Effect of Sexual Orientation. J Relig Health, 56, 2162-2179. https://doi.org/10.1007/s10943-017-0388-y 